\title{
On the Beta Function in Supersymmetric Gauge Theories
}

\author{
Giuseppe Carlino $^{1}$, Kenichi Konishi ${ }^{2}$, Nicola Maggiore $^{1}$ \\ and \\ Nicodemo Magnoli ${ }^{1}$ \\ Dipartimento di Fisica - Università di Genova ${ }^{(1)}$ \\ Istituto Nazionale di Fisica Nucleare - Sezione di Genova ${ }^{(1)}$ \\ Via Dodecaneso, 33 - 16146 Genova (Italy) \\ Dipartimento di Fisica - Università di Pisa ${ }^{(2)}$ \\ Istituto Nazionale di Fisica Nucleare - Sezione di Pisa ${ }^{(2)}$ \\ Via Buonarroti, 2, Ed. B. - 56127 Pisa (Italy) \\ E-mail: carlino@ge.infn.it; konishi@mailbox.dif.unipi.it; \\ maggiore@ge.infn.it; magnoli@ge.infn.it .
}

\begin{abstract}
We re-examine perturbative and nonperturbative aspects of the beta function in $N=1$ and $N=2$ supersymmetric gauge theories, make comments on the recent literature on the subject and discuss the exactness of several known results such as the NSVZ beta function.
\end{abstract}

GEF-TH 2/99

IFUP-TH 10/99

February, 1999 
1. In this note several comments on the beta function in supersymmetric gauge theories will be made in the light of the recent literature on the subject [1, 2, 3, 过. The bare Lagrangian of an $N=1$ supersymmetric gauge theory with generic matter content is given by

$$
L=\frac{1}{4} \int d^{2} \theta\left(\frac{1}{g_{h}^{2}(M)}\right) W^{a} W^{a}+\text { h.c. }+\int d^{4} \theta \sum_{i} \Phi_{i}^{\dagger} e^{2 V_{i}} \Phi_{i}
$$

where

$$
\frac{1}{g_{h}^{2}(M)}=\frac{1}{g^{2}(M)}+i \frac{\theta(M)}{8 \pi^{2}} \equiv \frac{\tau(M)}{4 \pi}
$$

and $g(M)$ and $\theta(M)$ stand for the bare coupling constant and vacuum parameter, $M$ being the ultraviolet cutoff. By a generalized nonrenormalization theorem [5] the effective Lagrangian at scale $\mu$ has the form,

$$
L=\frac{1}{4} \int d^{2} \theta\left(\frac{1}{g_{h}^{2}(M)}+\frac{b_{0}}{8 \pi^{2}} \log \frac{M}{\mu}\right) W^{a} W^{a}+\text { h.c. }+\int d^{4} \theta \sum_{i} Z_{i}(\mu, M) \Phi_{i}^{\dagger} e^{2 V_{i}} \Phi_{i},
$$

(plus higher dimensional terms). Here

$$
b_{0}=3 N_{c}-\sum_{i} T_{F i} ; \quad T_{F i}=\frac{1}{2} \quad \text { (quarks) } .
$$

Novikov et. al. used then the 1PI effective action to define a "physical" coupling constant for which they obtained the well known NVSZ beta function (Eq. 13. below) [6.

Recently the derivation of the NVSZ beta function was substantially clarified by Arkani-Hamed and Murayama [1, 2]. (See also [3].) They work entirely in the framework of the Wilsonian effective action (hence no subtleties due to zero momentum external lines, such as those leading to apparent violation of nonrenormalization theorem 5 , 母). They insist simply that at each infrared cutoff $\mu$ the matter kinetic terms be re-normalized so that it resumes the standard canonical form. Thus by introducing

$$
\Phi_{i}=Z_{i}^{-1 / 2} \Phi_{i}^{(R)},
$$

and by taking into account the appropriate anomalous Jacobian [8], one gets

$$
\begin{aligned}
L & =\frac{1}{4} \int d^{2} \theta\left(\frac{1}{g_{h}^{2}(M)}+\frac{b_{0}}{8 \pi^{2}} \log \frac{M}{\mu}-\sum_{i} \frac{T_{F}}{8 \pi^{2}} \log Z_{i}(\mu, M)\right) W^{a} W^{a}+\text { h.c. } \\
& +\int d^{4} \theta \sum_{i} \Phi_{i}^{(R) \dagger} e^{2 V_{i}} \Phi_{i}^{(R)} \\
& =\frac{1}{4} \int d^{2} \theta \frac{1}{g_{h}^{2}(\mu)} W^{a} W^{a}+\text { h.c. }+\int d^{4} \theta \sum_{i} \Phi_{i}^{(R) \dagger} e^{2 V_{i}} \Phi_{i}^{(R)} .
\end{aligned}
$$

where

$$
\frac{1}{g_{h}^{2}(\mu)} \equiv \frac{1}{g_{h}^{2}(M)}+\frac{b_{0}}{8 \pi^{2}} \log \frac{M}{\mu}-\sum_{i} \frac{T_{F i}}{8 \pi^{2}} \log Z_{i}(\mu, M) .
$$

This leads to the beta function (call it $\beta_{h}$ to distinguish it from the more commonly used definition):

$$
\beta_{h}\left(g_{h}\right) \equiv \mu \frac{d}{d \mu} \operatorname{Re} g_{h}=-\frac{g_{h}^{3}}{16 \pi^{2}}\left(3 N_{c}-\sum_{i} T_{F i}\left(1-\gamma_{i}\right)\right),
$$


where

$$
\gamma_{i}\left(g_{h}(\mu)\right)=-\mu \frac{d}{d \mu} \log Z_{i}(\mu, M)
$$

is the anomalous dimension of the $i$-th matter field. The same result follows by differentiating (7) with respect to $M$ with $\mu$ fixed, and by using $\gamma\left(g_{h}(M)\right)=+M \frac{d}{d M} \log Z_{i}(\mu, M)$.

The "holomorphic" coupling constant $g_{h}(\mu)$ is a perfectly good definition of the effective coupling constant: it is finite as $M \rightarrow \infty ; \quad \mu=$ finite, and physics below $\mu$ can be computed in terms of it. On the contrary, the coefficient of $W^{a} W^{a}$ in (3) is not a good definition of an effective coupling constant, as long as $N_{f} \neq 0$ : it is divergent in the limit the ultraviolet cutoff is taken to infinity. Let us note that, in spite of its name, the holomorphic coupling constant gets renormalized in a non-holomorphic way, due to the fact that $Z_{i}(\mu, M)$ is real.

Finally, in order to have the canonical form of gauge kinetic terms, $-F_{\mu \nu} F^{\mu \nu} / 4$, one must perform a further change of the variables,

$$
A_{\mu}=g_{c} A_{c \mu}
$$

and the corresponding rescaling of the gaugino field $\lambda_{\alpha}(x)$, to preserve supersymmetry. This introduces as functional-integral Jacobian an extra factor [2],

$$
\exp \frac{1}{4} \int d^{4} x \int d^{2} \theta \frac{N_{c} \log g_{c}^{2}}{8 \pi^{2}} W^{a} W^{a}+\text { h.c. }
$$

and as a consequence, leads to the change of the coupling constant 10,

$$
\operatorname{Re} \frac{1}{g_{h}^{2}}=\frac{1}{g_{c}^{2}}+\frac{N_{c}}{8 \pi^{2}} \log g_{c}^{2} .
$$

The NSVZ beta function [6] follows then from (12) and (8):

$$
\beta\left(g_{c}\right)=-\frac{g_{c}^{3}}{16 \pi^{2}} \frac{3 N_{c}-\sum_{i} T_{F i}\left(1-\gamma_{i}\right)}{1-N_{c} g_{c}^{2} / 8 \pi^{2}} .
$$

An important point of 2 is the fact that the Wilsonian coupling constant, whether "holomorphic" or "canonical", contains higher loop perturbative corrections in general; the often stated one-loop (perturbative) exactness of the Wilsonian effective coupling constant is valid only in particular cases, e.g., for the "holomorphic" coupling constant in the $N=1$ supersymmetric pure Yang-Mills theory.

2. The above procedure nicely "explains" the origin of the denominator of the NSVZ beta function. In the case of $N=1$ pure Yang-Mills theory the latter has led to an interesting conjecture [9]. However, it also leads to a new puzzle. In fact, the right hand side of (12) has a minimum at $g_{c}^{2}=8 \pi^{2} / N_{c}$, precisely corresponding to the pole of the NSVZ beta function, where it takes the value,

$$
\frac{N_{c}}{8 \pi^{2}} \log \frac{8 \pi^{2} e}{N_{c}}
$$

which is positive unless $N_{c}$ is rather large (i.e., unless $N_{c} \geq 215$ ). On the contrary the left hand side of (12) evolves down to zero if the beta function has no zero $\left(N_{f}<3 N_{c} / 2\right)$. Thus for large values of $g_{h}\left(g_{h}>8 \pi^{2} / N_{c} \log \left(8 \pi^{2} e / N_{c}\right)\right)$ the redefinition (12), with a real "canonical coupling constant", is not allowed. 
Since this problem occurs for any $N_{f}$ such that $N_{f}<3 N_{c} / 2$, let us for simplicity consider the case of the pure Yang Mills theory $\left(N_{f}=0\right)$ and compare the RG evolution in the two coupling constants. In this case, $\beta_{h}\left(g_{h}\right)$ is a pure one-loop effect, so that RG equation can be integrated in a closed form:

$$
\frac{1}{2 g_{h}^{2}(\mu)}-\frac{3 N_{c}}{16 \pi^{2}} \log \mu=\{\text { indep. of } \mu\} \equiv-\frac{3 N_{c}}{16 \pi^{2}} \log \Lambda,
$$

(which defines $\Lambda$ ) namely,

$$
\frac{1}{g_{h}(\mu)^{2}}=\frac{3 N_{c}}{8 \pi^{2}} \log \frac{\mu}{\Lambda}
$$

which evolves to the infrared and vanishes at $\mu=\Lambda$.

On the other hand, if one integrates

$$
\mu \frac{d}{d \mu} g_{c}=\beta\left(g_{c}\right)=-\frac{g_{c}^{3}}{16 \pi^{2}} \frac{3 N_{c}}{1-N_{c} g_{c}^{2} / 8 \pi^{2}},
$$

one gets

$$
\frac{1}{g_{c}^{2}(\mu)}+\frac{N_{c}}{8 \pi^{2}} \log g_{c}(\mu)^{2}-\frac{3 N_{c}}{8 \pi^{2}} \log \mu=\{\text { indep. of } \mu\} \text {. }
$$

so

$$
\frac{1}{g_{c}^{2}(\mu)}+\frac{N_{c}}{8 \pi^{2}} \log g_{c}(\mu)^{2}=\frac{1}{g_{h}(\mu)^{2}}=\frac{3 N_{c}}{8 \pi^{2}} \log \frac{\mu}{\Lambda},
$$

by using the same $\Lambda$ as in Eq.(16). The problem with (19) is that $g_{c}(\mu)$ does not "run" down to $\mu=\Lambda$; it runs only down to

$$
\mu_{0}=\frac{8 \pi^{2} e \Lambda}{N_{c}}>\Lambda
$$

(for $8 \pi^{2} e>N_{c}$ ), which corresponds to the pole of the NVSZ beta function.

Also, (19) apparently suggests the presence of another branch in which the coupling constant $g_{c}$ grows in the ultraviolet [9].

Actually both the absence of evolution below the scale $8 \pi^{2} e \Lambda / N_{c}$ and the apparent new phase of the theory are probably artefacts caused by the illegitimate change of variable (12). The pole at $g_{c}^{2}=8 \pi^{2} / N_{c}$ is then simply a sign of the failure of $g_{c}$ as a coupling constant (and $A_{c \mu}(x)$ as a functional variable), a sort of a singularity of parametrization, rather than of physics itself.

This however means that if one starts at high energies by using the standard "canonical" coupling constant and studies the RG evolution towards the low energies, one must switch to the "holomorphic" description at certain point (in any case, before the "critical" value $g_{c}^{2}=8 \pi^{2} / N_{c}$ is reached), in order to describe the physics smoothly down to $\mu=\Lambda$. The impossibility of writing a low energy effective Lagrangian with canonically normalized gauge kinetic terms, does not represent any inconsistency since the low energy physical degrees of freedom are in fact described by some gaugeinvariant composite fields, and not by gauge fields themselves. The latter fluctuate violently while the appropriate variables behave more smoothly. For $N_{f} \leq N_{c}$, the appropriate low energy degrees of freedom are mesonlike composite fields $M_{i j}=\tilde{Q}_{i} Q_{j}$; [1] for $N_{f}=N_{c}+1$ they are the mesons and

\footnotetext{
${ }^{1}$ As is well known SQCD with $N_{f}<N_{c}$ does not have well defined vacua if quarks are massless: we assume that all quark masses are small but nonvanishing. In the case $N_{f}=N_{c}=N$, the low energy degrees of freedom contains the baryon $B=\epsilon_{a_{1} a_{2} \ldots a_{N}} \epsilon^{i_{1} i_{2} \ldots i_{N}} Q_{i_{1}}^{a_{1}} Q_{i_{2}}^{a_{2}} \ldots Q_{i_{N}}^{a_{N}}$ and $\tilde{B}$ defined analogously in terms of $\tilde{Q}$ 's, as well.)
} 
"baryons"; for $N_{c}+1 \leq N_{f} \leq 3 N_{c}$ the low energy degrees of freedom are dual quarks and free mesons [11]. In particular, in the conformal window, i.e. for $3 N_{c} / 2 \leq N_{f} \leq 3 N_{c}$, the low energy theory admits two dual equivalent descriptions, one in terms of the original quarks and gluons of $S U\left(N_{c}\right)$ gauge theory, another in terms of $S U\left(N_{f}-N_{c}\right)$ gauge theory with $N_{f}$ flavors of dual quarks.

At larger values of $N_{f}\left(N_{f}>3 N_{c}\right)$ the low energy degrees of freedom are the original quarks and gluons, but since the theory is infrared free no obstruction arises against describing them by using the canonical coupling constant at all scales.

The success of the NSVZ beta function in the case of SQCD in the conformal window $\left(3 N_{c} / 2<\right.$ $N_{f}<3 N_{c}$ ) 11], especially the determination of the anomalous dimension of the matter fields,

$$
\gamma^{*}=\frac{3 N_{c}-N_{f}}{N_{f}},
$$

at the infrared fixed point, is consistent with the use of the holomorphic coupling constant (Eq.(8)). It does neither require the use of the canonical coupling constant nor necessitate the form of the original NSVZ beta function. This is important because the anomalous dimension at the IR fixed point is a physically observable number.

3. One might wonder how "exact" all this is. It is clear that the diagrammatic proof of the generalized nonrenormalization theorem of [5] is valid only within perturbation theory.

It was argued on the other hand in [2] that due to the existence of an anomalous $U_{R}(1)$ symmetry the beta functions are purely perturbative, hence the NSVZ beta function is exact perturbatively and nonperturbatively, at least for pure $N=1$ Yang-Mills theory. In fact, the (holomorphic) coupling constant at scale $M^{\prime}$ must satisfy

$$
\frac{8 \pi^{2}}{g_{h}^{2}\left(M^{\prime}\right)}=\frac{8 \pi^{2}}{g_{h}^{2}(M)}+f\left(\frac{8 \pi^{2}}{g_{h}^{2}(M)}, t\right)
$$

where $t \equiv \log \frac{M}{M^{r}}$ and $f$ is a holomorphic function of $g_{h}$. It follows that

$$
\beta_{h}\left(g_{h}\right)=\left.(d / d \log M) g_{h}(M)\right|_{g_{h}\left(M^{\prime}\right)}
$$

shares the same property. Together with the periodicity in $\theta$ with period $2 \pi$, one finds that

$$
\frac{d}{d t} \frac{8 \pi^{2}}{g_{h}^{2}(M)}=-\frac{16 \pi^{2}}{g_{h}^{3}} \beta_{h}\left(g_{h}\right)=\sum_{n=0}^{\infty} a_{n} e^{-8 \pi^{2} n / g_{h}^{2}},
$$

where $a_{n}$ is the $n$ - instanton contribution. Since the right hand side is independent of $\theta$ it must consists only of the perturbative term, $n=0$.

This argument is however only valid in theories in which $C P$ invariance is not spontaneously broken. Examples are the pure $N=1$ Yang-Mills theory or the $N=1$ SQCD at the origin of the space of vacua (with all scalar vevs vanishing): there the argument of [2] is valid and the use of the NSVZ beta function is justified.

Actually, the argument can be reversed and used in a stronger manner. In a generic point of the space of vacua of $N=1 \mathrm{SQCD}$, or of a pure $N=2$ supersymmetric Yang-Mills theory (a 
$N=1$ supersymmetric gauge theory with a matter chiral multiplet in the adjoint representation), for example, $C P$ invariance is spontaneously broken [13], and there is a nontrivial $\theta$ dependence. By holomorphic dependence of $\beta$ on $\tau=\frac{\theta}{2 \pi}+\frac{4 \pi i}{g^{2}}$ this implies that the beta function gets necessarily instanton corrections. A naïve application of the NSVZ formula to the $N=2$ pure Yang-Mills theory, which would simply yield the purely one-loop perturbative beta function " $\beta(g)$ " $=-g^{3} / 4 \pi^{2}$, is thus incorrect.?

4. It might be thought that in the $N=2$ gauge theories where the exact low-energy effective action is known [14, 15], the exact (nonperturbative) beta function can be computed. For such an attempts see [4]. For concreteness we restrict our discussion below to the simplest such case: the pure $N=2 S U(2)$ Yang-Mills theory.

Due to the holomorphic nature of Wilsonian effective action the RG equation can be cast into the form

$$
\beta(\tau) \equiv \mu \frac{d \tau}{d \mu}=\frac{2 i}{\pi}\left(1+c_{1} e^{2 \pi i \tau}+c_{2} e^{4 \pi i \tau}+\ldots\right)
$$

for $\operatorname{Im} \tau \gg 1$ (or $g^{2} \ll 1$ ) where

$$
\tau=\frac{\theta}{2 \pi}+\frac{4 \pi i}{g^{2}}
$$

and $\mu$ is the scale. Written separately for the real and imaginary parts, Eq.(25) reads [16, 12]:

$$
\begin{aligned}
& \beta_{g}(g, \theta) \equiv \mu \frac{d g}{d \mu}=-\frac{g^{3}}{4 \pi^{2}}\left(1+c_{1} \cos \theta e^{-8 \pi / g^{2}}+\ldots\right) ; \\
& \beta_{\theta}(g, \theta) \equiv \mu \frac{d \theta}{d \mu}=-4 c_{1} \sin \theta e^{-8 \pi / g^{2}}+\ldots
\end{aligned}
$$

The coupling constant and the $\theta$ parameter will evolve in the infrared up to the scale $\mu_{I R}$ which can be identified with the mass of the lightest charged particle. The difficulty in finding the beta function in these theories lies in the fact that in general the relation between $\mu_{I R}$ and the gauge invariant vev $u=\left\langle\operatorname{Tr} \phi^{2}\right\rangle$ is not simple. (For particular cases see below.) For this reason the knowledge of

$$
\tau_{e f f}(u)=\frac{d a_{D}}{d a}
$$

from the exact Seiberg-Witten solution as a function of the vacuum parameter $u$, is not sufficient to deduce the correct $\beta$ function.

By integrating Eq. (25) one gets:

$$
\int^{\tau} \frac{d \tau}{\beta(\tau)}-\log \mu=C
$$

where $C$ is a $\mu$-independent integration constant. The lower limit of the integration is left unspecified: to change it is equivalent to a shift of $C$ by a constant. We set now $\mu=M$ ( $M$ is the UV cutoff) and

\footnotetext{
${ }^{2}$ Note that the original derivation of the NSVZ beta function based on the calculation of certain one-instanton amplitude, applied in a simple-minded way to this theory, would yield the one-loop beta function. But this argument also fails since in the presence of the adjoint scalar vev, the standard instanton selection rules do not apply.
} 
use the known asymptotic behaviour of $\tau$ and $\beta(\tau)$. Since the theory at large $u$ is weakly coupled at all scales, one can use the known behaviour of $\tau_{\text {eff }}$ for such cases [17,

$$
\tau_{e f f} \simeq \frac{i}{\pi} \log \frac{4 a^{2}}{\Lambda^{2}}, \quad \theta_{0} \equiv-4 \operatorname{Arg} a
$$

where $\Lambda$ (real) is defined such that the massless monopole occurs at $u= \pm \Lambda^{2}$, to find the behavior of $\tau(M)$ as a function of $M$. In fact, by identifying $\mu_{I R}=M=\sqrt{2}|a|$, one gets

$$
\tau(M) \simeq \frac{\theta(M)}{2 \pi}+\frac{i}{\pi} \log \frac{2 M^{2}}{\Lambda^{2}} ; \quad \beta(\tau) \simeq \frac{2 i}{\pi}
$$

so that

$$
\begin{aligned}
C & =-\frac{i \pi}{2} \tau(M)-\log M+\text { const. } \\
& =-\frac{i \pi}{2}\left(\frac{\theta_{0}}{2 \pi}+\frac{i}{\pi} \log \frac{2 M^{2}}{\Lambda^{2}}\right)-\log M+\text { const. }=-\log \frac{e^{i \theta_{0} / 4}}{\Lambda}+\text { const. . }
\end{aligned}
$$

This shows how the dynamical mass scale $\Lambda$ and the bare $\theta\left(=\lim _{M \rightarrow \infty} \theta(M)\right)$ enter together as two integration constants of the RG equation.

In the strong coupling region, i.e. near $\tau=0$ (which is explored by theories near $u=\Lambda^{2}$ ) the infrared scale is given by $\mu_{I R}=\sqrt{2}\left|a_{D}\right|$ (the monopole mass). The divergent behavior of the second term of the left hand side of Eq. (29) must be cancelled by the first term:

$$
\int^{\tau} d \tau \frac{1}{\beta(\tau)} \simeq \log \frac{a_{D}}{\Lambda} .
$$

¿From the behavior of $a_{D}$ near $u=\Lambda^{2}$ [14]:

$$
\frac{a_{D}}{\Lambda} \simeq i \frac{u-\Lambda^{2}}{2 \Lambda^{2}}=16 i q_{D}=16 i e^{i \pi \tau_{D}},
$$

one finds

$$
\int^{\tau} d \tau \frac{1}{\beta(\tau)} \simeq i \pi \tau_{D}
$$

or (by using $\left.\tau=-1 / \tau_{D}\right)$ :

$$
\beta(\tau) \sim \frac{1}{i \pi \tau_{D}^{2}}=-\frac{i}{\pi} \tau^{2} \quad \text { as } \quad \tau \rightarrow 0 .
$$

For CP invariant cases $(\theta=0)$ this means the behavior

$$
\beta(g) \sim-\frac{2}{g},
$$

at large $g$.

The existence of the nontrivial space of vacua implies that, at any given scale, besides the usual parameters $g(\mu), \theta(\mu)$ one has the scale dependent vev,

$$
v(\mu)=\left\langle\operatorname{Tr} \phi^{2}(\mu)\right\rangle
$$

as another parameter of the theory. The usual moduli parameter $u$ is to be identified with its value in the low energy limit. Note that even though $v(\mu)$ is complex, its phase is related by anomaly to 
$\theta(\mu)$ so that only three parameters are independent. At the UV cutoff this relation is the standard one:

$$
v \rightarrow e^{i \alpha} v \Longleftrightarrow \theta \rightarrow \theta+2 \alpha
$$

so that only the combination $\theta_{\text {phys }}=\theta-2 \operatorname{Arg} v$ has a physical meaning.

The scale dependence of $v$ arises because in the instanton contributions to it the integrations over the collective coordinates must be done so that only the distances between $1 / M$ ( $M$ being the UV cutoff) and $1 / \mu$ are involved. For instance, in the one instanton contribution the integration over the instanton size must be limited to the region,

$$
\frac{1}{M} \leq \rho \leq \frac{1}{\mu}
$$

One is thus led to write one more RG equation besides Eq. (25):

$$
\mu \frac{d v}{d \mu}=2 v G(\tau)
$$

When the moduli parameter $u=\left\langle\operatorname{Tr} \phi^{2}\right\rangle$ is large as compared to $\Lambda^{2}$, the theory is weakly coupled at all scales, and $\sqrt{2}|a|$ can be taken as the lower cutoff $\mu$. At large $\mu$ (at $\tau \rightarrow i \infty)$ then

$$
G(\tau)=1-16 e^{2 i \pi \tau}+\ldots, \quad G<1
$$

from the known instanton expansion

$$
u(a)=a^{2} \sum_{n=0}^{\infty} b_{n}\left(\frac{\Lambda}{a}\right)^{4 n}, \quad \tau \simeq \frac{i}{\pi} \log \frac{4 a^{2}}{\Lambda^{2}}
$$

where $b_{0}=1 / 2, b_{1}=1 / 4$, etc.

Integrating Eq. (41) as before by using $d \mu / \mu=d \tau / \beta(\tau)$, one gets:

$$
2 \int^{\tau} \frac{d \tau}{\beta(\tau)} G(\tau)-\log \frac{8\left\langle\operatorname{Tr} \phi^{2}(\mu)\right\rangle}{\Lambda^{2}}=R,
$$

where $R$ is another $\mu$-independent integration constant (the factor $8 / \Lambda^{2}$ has been inserted for convenience). Again, by taking $\mu=M$ (large), using Eq. (31) and Eq. (42) as $\tau \rightarrow i \infty$, one gets

$$
R=-i \pi\left(\frac{\theta_{0}}{2 \pi}+\frac{i}{\pi} \log \frac{2 M^{2}}{\Lambda^{2}}\right)-\log \frac{8\left\langle\operatorname{Tr} \phi^{2}(M)\right\rangle}{\Lambda^{2}}=-\log \frac{4\left|\left\langle\operatorname{Tr} \phi^{2}(M)\right\rangle\right|}{M^{2}} .
$$

Note that $R$ is real: one finds thus the third integration constant $\frac{\left|\left\langle\operatorname{Tr} \phi^{2}(M)\right\rangle\right|}{M^{2}}$ besides $\theta_{0}$ and $\Lambda$. They are the free parameters of the theory.

By differentiating the left hand side of Eq.(44) with respect to $\tau$, one gets

$$
2 \frac{G(\tau)}{\beta(\tau)}-\frac{d v}{d \tau} \frac{1}{v}=0, \quad \text { or } \quad 2 v \frac{d \tau}{d v}=\frac{\beta(\tau)}{G(\tau)} .
$$

Going to the IR limit, i.e. $v \rightarrow u$, Eq. (46) reads

$$
2 u \frac{d \tau}{d u}=\frac{\beta(\tau)}{G(\tau)},
$$


where $\tau=\tau_{\text {eff }}$. But the left hand side, which is the derivative of the low-energy effective $\tau$ with respect to the vacuum parameter $u$, can be computed from the knowledge of the low energy actions only: the result is 邨

$$
2 u \frac{d \tau}{d u}=\frac{\beta(\tau)}{G(\tau)}=\frac{i}{\pi}\left(\frac{1}{\theta_{3}{ }^{4}}+\frac{1}{\theta_{4}{ }^{4}}\right) \equiv \tilde{\beta}(\tau)
$$

where $\theta_{i}(\tau)=\theta_{i}(0 \mid \tau)$, in terms of the standard elliptic theta functions [18].

$\tilde{\beta}(\tau)$ does not represent the nonperturbative beta function, in spite of the claim made in the literature to that effect, as can be seen from its behavior near $\tau=0$, for instance. ¿From the known properties of the theta functions

$$
\begin{aligned}
\frac{1}{\tilde{\beta}(\tau)} & =-i \pi \frac{\theta_{3}{ }^{4}\left(-1 / \tau_{D}\right) \theta_{4}{ }^{4}\left(-1 / \tau_{D}\right)}{\theta_{3}{ }^{4}\left(-1 / \tau_{D}\right)+\theta_{4}{ }^{4}\left(-1 / \tau_{D}\right)}=-i \pi\left(-i \tau_{D}\right)^{2} \frac{\theta_{3}{ }^{4}\left(\tau_{D}\right) \theta_{4}{ }^{4}\left(\tau_{D}\right)}{\theta_{3}{ }^{4}\left(\tau_{D}\right)+\theta_{4}{ }^{4}\left(\tau_{D}\right)} \\
& \simeq 16 \pi i \tau_{D}^{2} e^{2 \pi i \tau_{D}}, \quad \text { as } \quad \tau_{D} \rightarrow i \infty,
\end{aligned}
$$

hence

$$
\tilde{\beta}(\tau) \simeq-\frac{i}{16 \pi} \tau^{2} e^{-2 \pi i / \tau}
$$

which differs from the correct behavior of the beta function at $\tau \rightarrow 0+i \epsilon$, Eq. (36).

On the other hand, Eq. (50) is perfectly consistent with the behaviour of $\tau$ as a function of $u$.

$$
2 \int^{\tau} \frac{d \tau}{\beta(\tau)} G(\tau)=2 \int^{\tau} \frac{d \tau}{\tilde{\beta}(\tau)}=2 \int^{\tau_{D}} \frac{d \tau_{D}}{\tau_{D}{ }^{2}} \frac{1}{\tilde{\beta}(\tau)} \simeq 32 q_{D}=32 e^{i \pi \tau_{D}}
$$

¿From the expansion of $a(u)$ as $u \rightarrow \Lambda^{2}$ [17, one finds:

$$
\tau_{D}=-\frac{d a}{d a_{D}} \simeq-\frac{i}{\pi} \log \frac{u-\Lambda^{2}}{32 \Lambda^{2}} .
$$

Consequently:

$$
2 \int^{\tau} \frac{d \tau}{\beta(\tau)} G(\tau)=\text { const. }+\frac{u-\Lambda^{2}}{\Lambda^{2}}
$$

On the other hand, the second term of Eq. (44) gives

$$
-\log \frac{8 u}{\Lambda^{2}}=\text { const. }-\frac{u-\Lambda^{2}}{\Lambda^{2}}
$$

near $u=\Lambda^{2}$, so that the linear dependence on $u-\Lambda^{2}$ cancels out, as it should.

One can also check the behavior near $\tau=\tau_{0}=(1+i) / 2$ of Eq. (44), which corresponds to the infrared behavior of the theory near $u=0$. By a simple calculation using the results of Ritz $\llbracket$, one finds that:

$$
\frac{\beta(\tau)}{G(\tau)}=\tilde{\beta}(\tau) \simeq 2\left(\tau-\tau_{0}\right)
$$

Thus

$$
2 \int^{\tau} \frac{d \tau}{\beta(\tau)} G(\tau) \simeq \log \left(\tau-\tau_{0}\right)
$$

which is singular. But this singularity is expected because the second term of Eq. (44) is also logarithmically divergent, since:

$$
\left.u \simeq \pi i \theta_{3}^{4}(\tau)\right|_{\tau_{0}} \cdot\left(\tau-\tau_{0}\right)
$$


as can be shown by using the relation between $u$ and $\tau$ :

$$
u=\frac{\theta_{3}^{4}(\tau)+\theta_{4}^{4}(\tau)}{\theta_{3}^{4}(\tau)-\theta_{4}^{4}(\tau)} .
$$

Therefore, the logarithmic singularities cancel out.

These discussions simply check the derivation of (48), but also shows for instance that the zero of $\tilde{\beta}(\tau)=\beta(\tau) / G(\tau)$ at $\tau_{0}=(1+i) / 2$ must be attributed to a pole in the renormalization factor $G(\tau)$, not to a zero of the beta function. For if the beta function had a zero at $\tau_{0}=(1+i) / 2$ the first term of the right hand side of Eq.(29) would be singular there: such a singularity would have to be cancelled by the second term, which is however regular there because the infrared cutoff of the theory with $u=0$ is finite. This is another way of saying that the point $u=0$ is not a special point in the space of vacua: no restoration of the $S U(2)$ gauge symmetry occurs. The fact that $u \equiv\left\langle\operatorname{Tr} \phi^{2}\right\rangle=0$ there, is due to the instanton-induced renormalization of the composite operator $\operatorname{Tr} \phi^{2}$.

In conclusion, the problem of finding the correct nonperturbative beta function in supersymmetric gauge theories remains open. Let us also note that the related issue of the direct check of the SeibergWitten formulas in various $N=2$ gauge theories by direct instanton calculations, after the initial impressive success, leaves still many questions unanswered [19].

Ackowledgment One of the authors (K.K.) thanks M. Sakamoto, H. Murayama and F. Fucito for useful discussions at various stages of the work.

\section{References}

[1] N. Arkani-Hamed and H. Murayama, Phys. Rev. D57 (1998) 6638, hep-th/9705189.

[2] N. Arkani-Hamed and H. Murayama, hep-th/9707133.

[3] S. Arnone, C. Fusi and K. Yoshida, hep-th/9812022.

[4] J. A. Minahan and D. Nemeschansky, Nucl. Phys. B468 (1996) 72, hep-th/9601059; G. Bonelli and M. Matone, Phys. Rev. Lett. 76 (1996) 4107, hep-th/9602174; A. Ritz, Phys. Lett. B434 (1998) 54, hep-th/9710112; B. P. Dolan, Phys. Lett. B418 (1998) 107, hep-th/9710161; J. I. Latorre and C. A. Lutken, Phys. Lett. B421 (1998) 217, hep-th/9711150; A. Gorsky, A. Marshakov, A. Mironov, A. Morozov, Nucl.Phys. B527 (1998) 690, hep-th/9802007.

[5] V. A. Novikov, M. A. Shifman, A. I. Vainshtein and V. I. Zakharov, Phys. Lett. 157 B (1985) 169; ibid. 166B (1986) 329; M. A. Shifman and A. I. Vainshtein, Nucl. Phys. B 277 (1986) 456.

[6] V. A. Novikov, M. A. Shifman, A. I. Vainshtein and V. I. Zakharov, Nucl. Phys. B229 (1983) 381; ibid. B260 (1985) 157; Phys. Lett. B166 (1986) 334.

[7] D. R. T. Jones, Phys. Lett. 123B (1983) 45. 
[8] K. Konishi and K. Shizuya, Nuovo Cim. 90A (1985) 111.

[9] I. Kogan and M. Shifman, Phys. Rev. Lett. 75 (1995) 2085, hep-th/9504141.

[10] M. A. Shifman and A. I. Vainshtein, Nucl. Phys. B 359 (1991) 571.

[11] N. Seiberg, Nucl. Phys. B435 (1994) 484, hep-th/9411149.

[12] N. Seiberg, Phys. Lett. 206B (1988) 75.

[13] M. Di Pierro and K. Konishi, Phys. Lett B388 (96) 90.

[14] N. Seiberg and E. Witten, Nucl.Phys. B426 (1994) 19; Erratum ibid. B430 (1994) 485, hepth/9407087; N. Seiberg and E. Witten, Nucl. Phys. B431 (1994) 484, hep-th/9408099.

[15] A. Klemm, W. Lerche, S. Theisen and S. Yankielowicz, Phys. Lett. B344 (1995) 169, hepth/9411048; P. C. Argyres and A. F. Faraggi, Phys. Rev. Lett 74 (1995) 3931, hep-th/9411047; A. Hanany and Y. Oz, Nucl. Phys. B452 (1995) 283, hep-th/9505075; P. C. Argyres, M. R. Plesser and A. D. Shapere, Phys. Rev. Lett. 75 (1995) 1699, hep-th/9505100.

[16] V. G. Knizhnik and A. Yu. Morozov, JETP Lett. 39 (1984) 241.

[17] F. Ferrari and A. Bilal, Nucl. Phys. B469 (1996) 387, hep-th/9602082.

[18] E. T. Whittaker and G. N. Watson, "A Course of Modern Analysis", Cambridge University Press, Fourth Ed. (1927).

[19] N. Dorey, V. V. Khoze and M. P. Mattis, Nucl. Phys. B492 (1997) 607, hep-th/9612231; Phys. Lett. B396 (1997) 141, hep-th/9611016, and references therein. 